\title{
Oblique Stagnation-Point Darcy Flow towards a Stretching Sheet
}

\author{
P. Singh ${ }^{1 \dagger}$, D. Sinha ${ }^{2}$ and N.S. Tomer ${ }^{3}$ \\ ${ }^{1}$ ITM University, Sector-23A, Gurgaon, Haryana-122017 \\ ${ }^{2}$ Centre for Mathematical Sciences (CMS), Banasthali University, Rajasthan-304022, India \\ ${ }^{3}$ Faculty of Mathematics, Department of Higher Education, Haryana, India \\ †Corresponding Author Email: phool.singh24@gmail.com \\ (Received May 23, 2010; accepted January 5, 2011)
}

\begin{abstract}
An attempt is made for the study of steady two-dimensional flow of a viscous and incompressible fluid striking at some angle of incidence on a stretching sheet. Fluid is considered in the porous media obeying Darcy law, in the presence of radiation effect. Rosseland approximation is use to model the radiative heat transfer. The stream function splits into a Hiemenz and a tangential component. Using similarity variables, the governing partial differential equations are transformed into a set of three non-dimensional ordinary differential equations. These equations are then solved numerically using fifth order Runge-Kutta Fehlberg method with shooting technique. In the present reported work the effects of striking angle, radiation parameter, porosity parameter and the Prandtl number on flow and heat transfer characteristics have been discussed. Variations of above discussed parameters with the stretching sheet parameter have been graphically presented.
\end{abstract}

Keywords: Oblique Stagnation-point, Heat transfer, Stretching sheet, Porous media.

\section{NOMENCLATURE}

\begin{tabular}{ll}
$A$ & \multicolumn{1}{l}{ Porosity parameter } \\
$a, b, c, \alpha \quad$ are some positive constant \\
$C_{p} \quad$ & specific heat at constant pressure \\
$f, g$ & dimensionless primary and secondary flow, \\
& respectively \\
$\mathrm{k}$ & permeability of saturated porous media \\
$K$ & thermal Conductivity \\
$k_{1}$ & mean absorption coefficient \\
$p$ & pressure of fluid \\
$\mathrm{Pr}$ & Prandtl number \\
$q_{r}$ & radiative heat flux \\
$R$ & radiation parameter \\
$T$ & temperature profile \\
$T_{\infty}$ & constant temperature of the fluid far away \\
& from sheet
\end{tabular}

\section{INTRODUCTION}

The flow of an incompressible viscous fluid over a stretching surface is important in various processes. In technical processes concerning extrusion of polymer sheets, rolling and manufacturing plastic films and artificial fibers, involves the drawing of strips. Strips which are extruded from a die through a moving fluid with controlled cooling system may become sometime stretched. The stretching surfaces undergo cooling/heating that causes surface velocity and
$T_{w} \quad$ temperature of stretching sheet

$u \quad$ velocity component along $\mathrm{x}$-axis

$u_{w} \quad$ velocity of stretching sheet

$v \quad$ velocity component along $\mathrm{y}$-axis

$\sigma \quad$ Stefan's-Boltzmann constant

$\theta \quad$ dimensionless temperature

$\psi \quad$ stream function

$v \quad$ kinematic viscosity

$\gamma \quad$ striking angle of the fluid

$\eta, \xi \quad$ similarity variables

$\lambda$ stretching sheet parameter

$\tau_{w} \quad$ shear stress

$\xi_{s} \quad$ stagnation point. temperature variations. In stagnation point flow, a rigid wall or a stretching surface occupies the entire horizontal $\mathrm{x}$-axis. The fluid domain is $y>0$ and the flow strikes on the stretching surface either orthogonal or at some angle of incidence. This simple model of oblique stagnation point would enable us to understand how a boundary layer begins to develop. Therefore, location of stagnation point is of great importance to analyze behavior of boundary layer. Present research field has attracted many researchers in recent years due to its astounding applications. Crane (1970) studied the 
steady two-dimensional flow of a viscous and incompressible fluid induced by the stretching of an elastic flat sheet in its own plane with a velocity varying linearly with the distance from a fixed point due to the application of a uniform stress. Gupta and Mahapatra (2003), and Sharma and Singh (2009) analyzed stagnation-point flow towards a stretching surface. They reported that a boundary layer is formed when stretching velocity is less than the free stream velocity. As the stretching velocity exceeds the free stream velocity than an inverted boundary layer is formed. Singh et al. (2011) reported effect of porosity parameter and radiations on stretching sheet for orthogonal flow. Reza and Gupta (2005) analyzed oblique stagnation-point flow towards a stretching surface.

Lok et al. (2006-2007) investigated the non-orthogonal stagnation-point for Newtonian and non-Newtonian flows towards a stretching sheet. Numerical solutions of the similarity equation are obtained in details. They found that the position of stagnation point depend on stretching sheet parameter and angle of incidence. Labropulu et al. (2009, 2010) analyzed oblique stagnation point flow of incompressible visco-elastic fluid towards a stretching sheet. Tilley and Weidman (1998) studied interaction between two planar oblique stagnation-point flows of different immiscible fluid.

Flow through porous media plays an important role in many practical applications such as ground water flows, extrusion of a polymer sheet from a dye, enhanced oil recovery processes and pollution movement. Attia (2007) discussed the effect of porosity parameter on the velocity and thermal boundary layer. Elbashbeshy and Bazid (2009) studied heat transfer in porous medium. Rosali et al. (2011) studied stagnation point flow and heat transfer over a stretching/shrinking sheet in a porous medium. They indicate that dual solutions exist for the shrinking case. Hayat et al. (2010) use Homotopy Analysis Method (HAM) to give analytic solution for flow through porous medium. All authors discuss above have taken Darcy flow. Pal and Modal (2010) discussed non-Darcian flow in porous medium.

The study of heat transfer and flow field is necessary for determining the quality of the final product. In technological processes at high temperature, thermal radiation effect cannot be neglected. Free convection heat transfer with radiation effect near the isothermal stretching sheet near the stagnation point has been investigated by Ghaly and Elbarbary (2002). Pop et al. (2004) studied radiation effect over a flat plate near stagnation point. They found that a boundary layer thickness increases with radiation. El-Aziz (2009) investigated radiation effects for the case of unsteady stretching sheet. Amaouche and Boukari (2003) studied the influence of thermal convection on non-orthogonal stagnation point flow. In certain porous media applications such as those involving heat removals from nuclear fuel debris, underground disposal of radiative waste material, storage of food stuffs, the study of heat transfer is of much importance.

The flow in the porous media deals with the analysis in which the differential equation governing the fluid motion is based on the Darcy's law which accounts for the drag exerted by the porous medium. The authors in the present manuscript studied the steady twodimensional flow of a viscous incompressible fluid striking at different angles of incidence on a stretching sheet. Fluid is considered in porous medium in the presence of radiation effect. Rosseland approximation is use to model the radiative heat transfer. To the best of author's knowledge, this problem has not been discussed so far.

\section{Formulation of the Problem}

Consider steady two-dimensional flow of a viscous incompressible fluid striking at some angle of incidence $\gamma$ on a stretching sheet. Fluid is considered in the porous medium obeying Darcy law in the presence of radiation effect. The stretching sheet has uniform temperature $T_{w}$ and linear velocity $u_{w}$. Stretching sheet is placed in the plane $y=0$ and $x$-axis is taken along the sheet. The fluid occupies the upper half plane i.e. $y \geq 0$ as shown in Fig. 1.

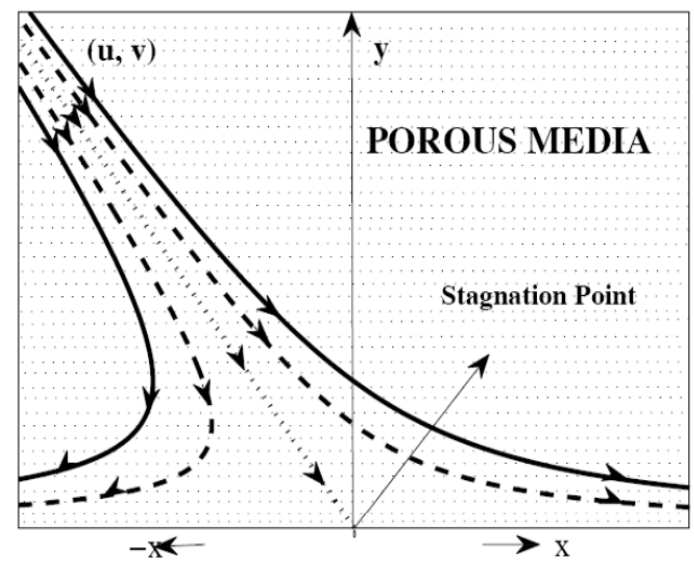

Fig. 1. Physical model.

It is assumed that external field is zero. The governing equations of continuity, momentum and energy under above assumptions are given by

$\frac{\partial u}{\partial x}+\frac{\partial v}{\partial y}=0$

$u \frac{\partial u}{\partial x}+v \frac{\partial u}{\partial y}=-\frac{1}{\rho} \frac{\partial p}{\partial x}+v\left(\frac{\partial^{2} u}{\partial x^{2}}+\frac{\partial^{2} u}{\partial y^{2}}\right)-\frac{v u}{k}$,

$u \frac{\partial v}{\partial x}+v \frac{\partial v}{\partial y}=-\frac{1}{\rho} \frac{\partial p}{\partial y}+v\left(\frac{\partial^{2} v}{\partial x^{2}}+\frac{\partial^{2} v}{\partial y^{2}}\right)-\frac{w}{k}$,

$\rho C_{p}\left(u \frac{\partial T}{\partial x}+v \frac{\partial T}{\partial y}\right)=K \frac{\partial^{2} T}{\partial y^{2}}-\frac{\partial q_{r}}{\partial y}$

where $u$ and $v$ are velocity components along $x$ and $y$ axes respectively, $v$ is kinematic viscosity, $k$ is permeability of saturated porous medium, $T$ is the temperature, $\rho$ is density of the fluid, $K$ is thermal conductivity and $C_{p}$ is specific heat at constant pressure. Boundary conditions are, 


$$
\left.\begin{array}{l}
y=0: \quad u=u_{w}(x)=c x, v=0, T=T_{w} \\
y \rightarrow \infty: u=a x \sin \gamma+b y \cos \gamma, v=-a y \sin \gamma, T=T_{\infty} .
\end{array}\right\}
$$

This flow model is called favorable flow for $0 \leq \gamma<\pi / 2$ and unfavorable flow for $\pi / 2<\gamma \leq \pi$. Case $\gamma=\pi / 2$, is known as orthogonal flow.

\section{Method OF Solution}

Eliminating pressure $p$ term from Eqs. (2) and (3), we have

$$
\begin{aligned}
\frac{\partial u}{\partial y} \frac{\partial u}{\partial x}+u & \frac{\partial^{2} u}{\partial y \partial x}+\frac{\partial v}{\partial y} \frac{\partial u}{\partial y}+v \frac{\partial^{2} u}{\partial y^{2}}=\frac{\partial u}{\partial x} \frac{\partial v}{\partial x}+u \frac{\partial^{2} v}{\partial x^{2}} \\
+ & \frac{\partial v}{\partial y} \frac{\partial v}{\partial x}+v \frac{\partial^{2} v}{\partial x \partial y}-\frac{v}{k}\left(\frac{\partial u}{\partial y}+\frac{\partial v}{\partial x}\right) \\
+ & v\left(\frac{\partial^{3} u}{\partial y \partial x^{2}}+\frac{\partial^{3} u}{\partial y^{3}}-\frac{\partial^{3} v}{\partial x^{3}}-\frac{\partial^{3} v}{\partial x \partial y^{2}}\right)
\end{aligned}
$$

Introducing the dimensionless variables $\xi=\sqrt{c / v} x, \eta=\sqrt{c / v} y$ and the stream function $\psi(\xi, \eta)$ as defined by $u=\partial \psi / \partial \eta$ and $v=-\partial \psi / \partial \xi$.

We seek solution of Eq. (6) in the form of $\psi=\xi f(\eta)+g(\eta)$, where the function $f(\eta)$ and $g(\eta)$ are referring to the normal and tangential component of the flow. Therefore, velocity components are given by,

$$
u(\xi, \eta)=\xi f^{\prime}(\eta)+g^{\prime}(\eta) \text { and } v(\xi, \eta)=-f(\eta) .
$$

Equation of Continuity (1) is identically satisfied by $u(\xi, \eta)$ and $v(\xi, \eta)$ defined as above and Eq. (6) reduces to,

$$
\begin{gathered}
f^{\prime}(\eta)\left(\xi f^{\prime \prime}(\eta)+g^{\prime \prime}(\eta)\right)+\left(\xi f^{\prime}(\eta)+g^{\prime}(\eta)\right) f^{\prime \prime}(\eta)- \\
f^{\prime}(\eta)\left(\xi f^{\prime \prime}(\eta)+g^{\prime \prime}(\eta)\right)-f(\eta)\left(\xi f^{\prime \prime \prime}(\eta)+g^{\prime \prime \prime}(\eta)\right) \\
=\left(\xi f^{\prime \prime \prime \prime}(\eta)+g^{\prime \prime \prime \prime}(\eta)\right)-A\left(\xi f^{\prime \prime}(\eta)+g^{\prime \prime}(\eta)\right) .
\end{gathered}
$$

Here $A(=v / c k)$ is porosity parameter. Comparing coefficient of $\xi$, we get,

$$
\begin{gathered}
f^{\prime \prime \prime \prime}(\eta)+f^{\prime}(\eta) f^{\prime \prime}(\eta)+f(\eta) f^{\prime \prime \prime}(\eta)-2 f^{\prime}(\eta) f^{\prime \prime}(\eta) \\
-A f^{\prime \prime}(\eta)=0 \\
g^{\prime \prime \prime \prime}(\eta)+f^{\prime}(\eta) g^{\prime \prime}(\eta)+f(\eta) g^{\prime \prime \prime}(\eta)-f^{\prime}(\eta) g^{\prime \prime}(\eta) \\
-g^{\prime}(\eta) f^{\prime \prime}(\eta)-A g^{\prime \prime}(\eta)=0
\end{gathered}
$$

Equations (7) and (8) can be rewritten as,

$$
\begin{gathered}
f^{\prime \prime \prime}(\eta)+f(\eta) f^{\prime \prime}(\eta)-\left(f^{\prime}(\eta)\right)^{2}-A f^{\prime}(\eta)+C_{1}=0 \\
g^{\prime \prime \prime}(\eta)+f(\eta) g^{\prime \prime}(\eta)-f^{\prime}(\eta) g^{\prime}(\eta)-A g^{\prime}(\eta) \\
+C_{2}=0
\end{gathered}
$$

where $C_{1}$ and $C_{2}$ are constant of integration to be determined by using the boundary conditions:

$$
\left.\begin{array}{ll}
f(0)=0, & f^{\prime}(0)=1, \quad f^{\prime}(\infty)=\lambda \sin \gamma \\
g(0)=0, & g^{\prime}(0)=0, \quad g^{\prime \prime}(\infty)=r \cos \gamma
\end{array}\right\}
$$

Using boundary condition (11) in Eqs. (9) and (10), we get,

$$
\begin{array}{r}
f^{\prime \prime \prime}(\eta)+f(\eta) f^{\prime \prime}(\eta)-\left(f^{\prime}(\eta)\right)^{2}-A\left(f^{\prime}(\eta)-\lambda \sin \gamma\right) \\
-(\lambda \sin \gamma)^{2}=0 \\
g^{\prime \prime \prime}(\eta)+f(\eta) g^{\prime \prime}(\eta)-f^{\prime}(\eta) g^{\prime}(\eta)-\alpha r \cos \gamma \\
-A\left(g^{\prime}(\eta)-r \eta \cos \gamma\right)=0 .
\end{array}
$$

where $\lambda=c / a$ is stretching parameter and $r=c / b$ is secondary stretching parameter in normal direction.

Radiative heat flux $q_{r}$ in governing boundary layer equation of energy (4) is approximated by Rosseland approximation, which gives

$$
q_{r}=-\frac{4 \sigma}{3 k_{1}} \frac{\partial T^{4}}{\partial y}
$$

It is assumed that the temperature difference within the flow is so small that $T^{4}$ can be expressed as a linear function of $T_{\infty}$. This can be obtained by expanding $T^{4}$ in a Taylor series about $T_{\infty}$ and neglecting the higher order terms. Thus we get, $T^{4} \cong 4 T_{\infty}{ }^{3} T-3 T_{\infty}{ }^{4}$. Heat is transferred by forced convection which involve the only normal component of flow field, therefore $\theta(\eta)$ is dimensionless temperature defined as $\theta(\eta)=\left(T-T_{\infty}\right) /\left(T_{w}-T_{\infty}\right)$. Using this in energy Eq. (4), we have

$$
\theta^{\prime \prime}(\eta)\left\{1+\frac{4 R}{3}\right\}+\operatorname{Pr} \theta^{\prime}(\eta) f(\eta)=0
$$

where $R$ is radiation parameter $\left(4 \sigma T_{\infty}^{3} / K k_{1}\right)$ and $\operatorname{Pr}$ is Prandtl number $\left(\mu C_{p} / K\right)$, with boundary conditions

$$
\theta(0)=1, \quad \theta(\infty)=0 .
$$

In the absence of an analytical solution of a problem, a numerical solution is indeed an obvious and natural choice. Thus, the governing boundary layer and thermal boundary layer Eqs. (12-14) with boundary conditions (11) and (15), are solved using fifth order Runge-Kutta Fehlberg method with shooting technique. A physical quantity of interest is the skin friction, or shear stress $\tau_{w}$ at the wall, which is defined as

$$
\begin{aligned}
& \tau_{w}=\mu\left(\frac{\partial u}{\partial x}+\frac{\partial u}{\partial y}\right)_{y=0} \\
& \tau_{w}=\mu\left(\xi f^{\prime \prime}(0)+g^{\prime \prime}(0)\right)
\end{aligned}
$$

The dividing streamlines $\psi=0$ and the curve $u=0$ intersect the wall at the stagnation point where $\tau_{w}=0$, hence the location of stagnation point $\xi_{S}$ is given by,

$$
\xi_{s}=-\frac{g "(0)}{f "(0)}
$$

\section{NUMERICAL SIMULATION}

The set of non-linear coupled differential Eqs. (12), (13) and (14) subject to the boundary conditions (11) 
Table 1. Comparision of value of $f^{\prime \prime}(0)$ for different values of $\lambda$

\begin{tabular}{|l|c|c|c|c|c|}
\hline \multicolumn{7}{|c|}{ Value of $f^{\prime \prime}(0)$ for different $\lambda$ for $\gamma=\pi / 2, A=0$} \\
\hline Value of $\lambda$ & Present paper & $\begin{array}{l}\text { Lok et al. } \\
(2006)\end{array}$ & $\begin{array}{l}\text { Gupta and Mahapatra } \\
(2003)\end{array}$ & $\begin{array}{l}\text { Pop et al. } \\
(2004)\end{array}$ & $\begin{array}{l}\text { Singh et al. } \\
(2011)\end{array}$ \\
\hline$\lambda=0.1$ & -0.976371 & -0.969388 & -0.9694 & -0.9694 & -0.976371 \\
\hline$\lambda=0.2$ & -0.921594 & -0.918110 & -0.9181 & -0.9181 & -0.921592 \\
\hline$\lambda=0.5$ & -0.667686 & -0.667271 & -0.6673 & -0.6673 & -0.667685 \\
\hline$\lambda=2$ & 2.0174763 & 2.017615 & 2.0175 & 2.0174 & 2.0174765 \\
\hline$\lambda=3$ & 4.7290669 & 4.729694 & 4.7293 & 4.7290 & 4.7290671 \\
\hline
\end{tabular}

Table 2. Values of $f^{\prime \prime}(0)$ for different values of stretching parameter $\lambda, A$ and $\gamma$.

\begin{tabular}{|c|c|c|c|c|c|c|}
\hline \multicolumn{9}{|c|}{ Value of $f^{\prime \prime}(0)$ for different $\lambda, A$ and $\gamma$} \\
\hline $\begin{array}{c}\text { Value of } \\
\lambda\end{array}$ & \multicolumn{2}{|c|}{$\gamma=\pi / 3$} & \multicolumn{2}{c|}{$\gamma=\pi / 4$} & \multicolumn{2}{c|}{$\gamma=\pi / 5$} \\
\cline { 2 - 7 } & $A=0$ & $A=3$ & $A=0$ & $A=3$ & $A=0$ & $A=3$ \\
\hline$\lambda=0.1$ & -0.98245903 & -1.85670878 & -0.98928540 & -1.88347580 & -0.976371 & -0.976371 \\
\hline$\lambda=0.2$ & -0.93785336 & -1.70726572 & -0.95565382 & -1.76280961 & -0.921594 & -0.921594 \\
\hline$\lambda=0.5$ & -0.73512206 & -1.22387716 & -0.80740039 & -1.37706387 & -0.667686 & -0.667686 \\
\hline$\lambda=2$ & 1.40094697 & 1.88577538 & 0.73844740 & 1.02742229 & 0.29454561 & 0.42244459 \\
\hline$\lambda=3$ & 3.56624221 & 4.50668261 & 2.31296994 & 3.01463037 & 1.47033096 & 1.97345837 \\
\hline
\end{tabular}

Table 3. Values of $g^{\prime \prime}(0)$ for different values of stretching parameter $\lambda, A$ and $\gamma$.

\begin{tabular}{|c|c|c|c|c|c|c|}
\hline \multicolumn{7}{|c|}{ Value of $g^{\prime \prime}(0)$ for different $\lambda, A$ and $\gamma$} \\
\hline $\begin{array}{c}\text { Value of } \\
\lambda\end{array}$ & \multicolumn{2}{|c|}{$\gamma=\pi / 3$} & \multicolumn{2}{c|}{$\gamma=\pi / 4$} & \multicolumn{2}{c|}{$\gamma=\pi / 5$} \\
\cline { 2 - 7 } & $A=0$ & $A=3$ & $A=0$ & $A=3$ & $A=0$ & $A=3$ \\
\hline$\lambda=0.1$ & -0.33909700 & 2.31168374 & -0.48986521 & 3.27346165 & -0.56965453 & 3.74889368 \\
\hline$\lambda=0.2$ & -0.30381001 & 2.29538669 & -0.44688114 & 3.25460592 & -0.52695451 & 3.73093626 \\
\hline$\lambda=0.5$ & -0.22593960 & 2.24744169 & -0.34861407 & 3.19884865 & -0.42652725 & 3.67765080 \\
\hline$\lambda=2$ & -0.05336530 & 2.04001724 & -0.11252517 & 2.94932616 & -0.16791715 & 3.43320628 \\
\hline$\lambda=3$ & -0.00331194 & 1.93127333 & -0.03941102 & 2.81226684 & -0.08264740 & 3.29385003 \\
\hline
\end{tabular}

and (15) constitute a two-point boundary value problem. In order to solve these equations numerically, we follow most efficient numerical shooting technique with fifth-order Runge-Kutta-Fehlberg integration scheme. In this method it is most important to choose the appropriate finite values of $\eta \rightarrow \infty$. The solution process is repeated with another large value of $\eta_{\infty}$ until two successive values of $f^{\prime \prime}(0)$ and $\theta^{\prime}(0)$ differ only after a desired digit signifying the limit of the boundary along $\eta$. The last value of $\eta_{\infty}$ is chosen as appropriate value of the limit $\eta \rightarrow \infty$ for that particular set of parameters. The three ordinary differential Eqs. (12), (13) and (14) were first formulated as a set of eight first-order simultaneous equations. To solve this system we require eight initial conditions whilst we have only five initial conditions $f(0)$ and $f^{\prime}(0)$ on $f(\eta), g(0)$ and $g^{\prime}(0)$ on $g(\eta)$, and one initial condition $\theta(0)$ on $\theta(\eta)$. Still there are three initial conditions $f^{\prime \prime}(0), \quad g^{\prime \prime}(0)$ and $\theta^{\prime}(0)$ which are not prescribed.

However the values of $f^{\prime}(\eta), g^{\prime \prime}(\eta)$ and $\theta(\eta)$ are known at $\eta \rightarrow \infty$. Now we employ the numerical shooting technique where these three ending boundary conditions are utilized to produce three known initial conditions at $\eta=0$. Finally, the problem has been solved numerically using fifth-order Runge-Kutta Fehlberg method.

\section{RESUlts AND Discussion}

Fifth order Runge-Kutta Fehlberg method with the help of shooting method is used to solve Eqs. (12)-(14) for different values of $\lambda, R, \operatorname{Pr}, \gamma$ and $A$ taking step size 0.001 . While numerical simulation, step size 0.002 and 0.003 were all checked and values of $f^{\prime \prime}(0), g^{\prime \prime}(0)$ and $\theta^{\prime}(0)$ were found in each case correct up to six decimal places. Hence the scheme used in this paper stable and accurate. We have compared values of $f^{\prime \prime}(0)$ for orthogonal flow $\gamma=\pi / 2$ with Lok et al. (2006), Gupta and Mahapatra (2003), Singh et al. (2011) and Pop et al. (2004) as shown in Table 1. Physically, positive sign of $f^{\prime \prime}(0)$ implies that the fluid exerts a drag force on the sheet and negative sign implies the opposite. The streamline patterns for the orthogonal and oblique flows are shown in Figs. 2(a-e) for $A=0$. Both the favorable and unfavorable cases are considered. 


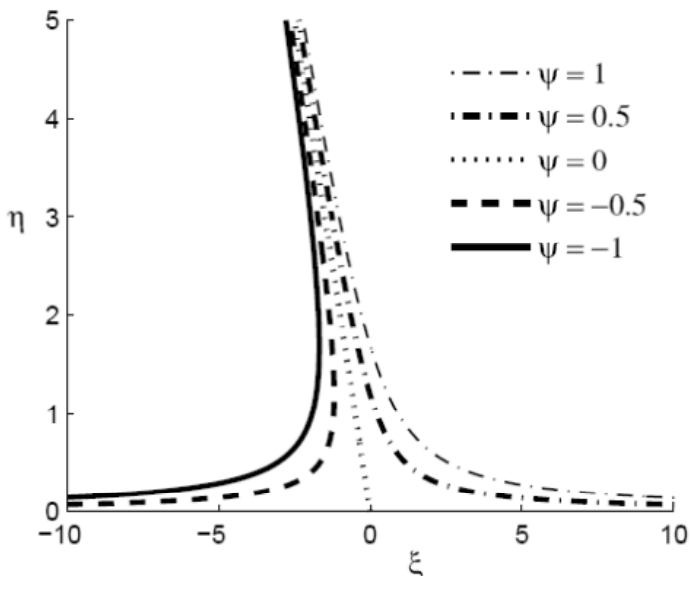

Fig. 2a). Streamline pattern for oblique flow when $\gamma=\pi / 4$, taking $A=0$.

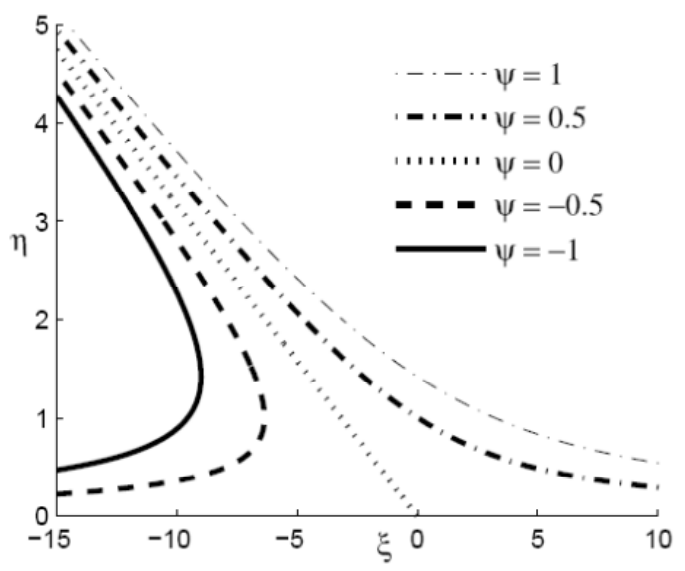

Fig. 2b). Streamline pattern for oblique flow when $\gamma=\pi / 20$, taking $A=0$.

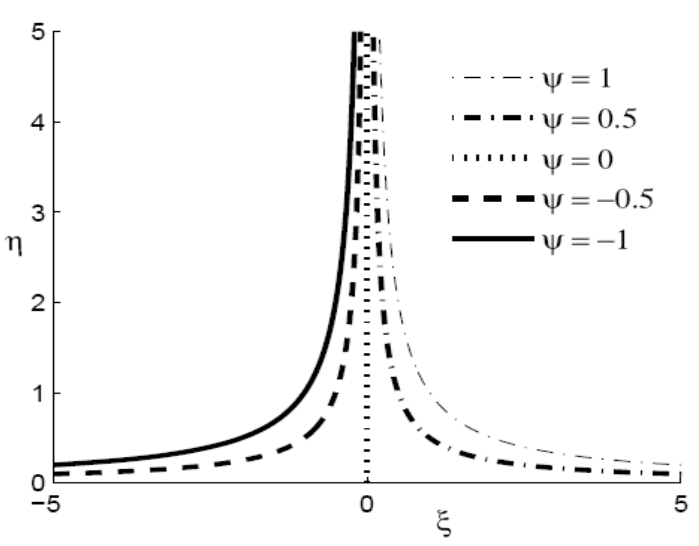

Fig. 2e). Streamline pattern for oblique flow when $\gamma=\pi / 2$, taking $A=0$.

Figure 3 shows that the boundary layer thickness decreases considerably as $\lambda$ increases at the points where $f^{\prime}(\eta)$ reaches the boundary condition. The increase in the value of $\lambda$ implies that free stream velocity increases in comparison to stretching velocity, which results in the increase in pressure and straining

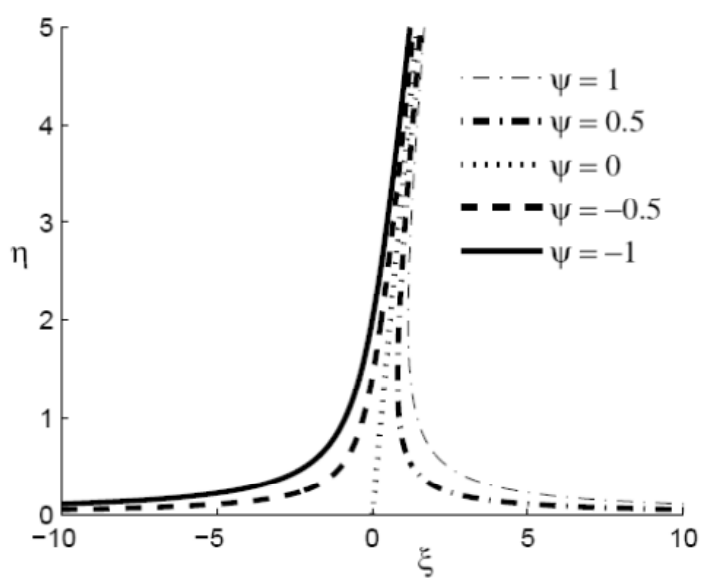

Fig. 2c). Streamline pattern for oblique flow when $\gamma=2 \pi / 3$, taking $A=0$.

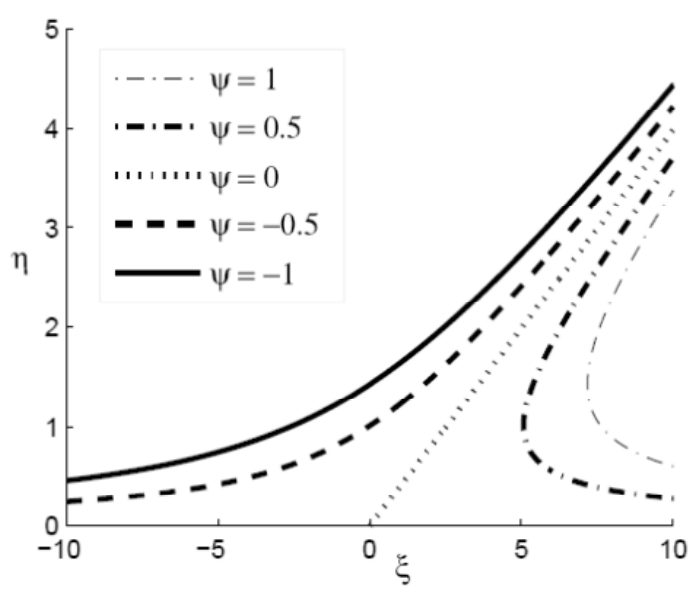

Fig. 2d). Streamline pattern for oblique flow when $\gamma=15 \pi / 16$, taking $A=0$.

motion near stagnation point and hence thinning of boundary layer takes place.

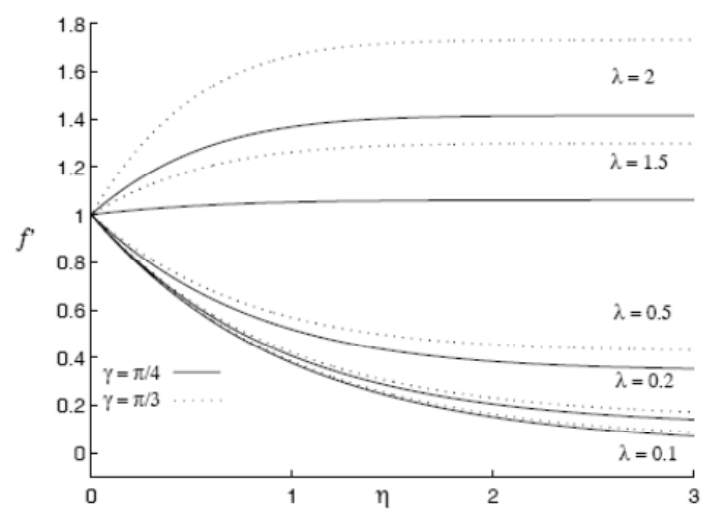

Fig. 3. Velocity profile $f^{\prime}(\eta)$ versus $\eta$ for different values $\lambda, \gamma=\pi / 4, \pi / 3$ and $A=0$.

The phenomenon of thinning of boundary layer thus implies increased shear stress at the sheet, which is also seen in Table 2. The streamline $\psi=0$ meets the wall at $\xi_{s}$ where $\xi_{s}$ is the point of stagnation and zero skin 
Table 4. Location of stagnation point $\xi_{s}$ for different values of $\lambda, A$ and $\gamma$.

\begin{tabular}{|c|c|c|c|c|c|c|}
\hline \multicolumn{7}{|c|}{ Value of $\xi_{s}$ for different $\lambda, A$ and $\gamma$} \\
\hline $\begin{array}{c}\text { Value of } \\
\lambda\end{array}$ & \multicolumn{2}{|c|}{$\gamma=\pi / 3$} & \multicolumn{2}{c|}{$\gamma=\pi / 4$} & \multicolumn{2}{c|}{$\gamma=\pi / 5$} \\
\cline { 2 - 7 } & $A=0$ & $A=3$ & $A=0$ & $A=3$ & $A=0$ & $A=3$ \\
\hline$\lambda=0.1$ & -0.3452 & 1.2450 & -0.4952 & 1.7380 & -0.5730 & 1.9695 \\
\hline$\lambda=0.2$ & -0.3239 & 1.3445 & -0.4676 & 1.8463 & -0.5444 & 2.0682 \\
\hline$\lambda=0.5$ & -0.3073 & 1.8363 & -0.4318 & 2.3228 & -0.4983 & 2.4697 \\
\hline$\lambda=2$ & 0.03810 & -1.0818 & 0.1524 & -2.8706 & 0.5701 & -8.1270 \\
\hline$\lambda=3$ & 0.00092 & -0.4285 & 0.0170 & -0.9329 & 0.0562 & -1.6691 \\
\hline
\end{tabular}

Table 5. Values of rate of heat transfer for different values of stretching sheet parameter $\lambda, A$, striking angle $\gamma$ taking radiation parameter $R=1$ and Prandtl number $\operatorname{Pr}=0.71$.

\begin{tabular}{|c|l|l|l|l|l|l|}
\hline \multicolumn{7}{|c|}{ Value of $-\theta^{\prime}(0)$ for different $\lambda, A$ and $\gamma$} \\
\hline $\begin{array}{c}\text { Value of } \\
\lambda\end{array}$ & \multicolumn{1}{|c|}{$\gamma=\pi / 2, \operatorname{Pr}=0.71, R=1$} & \multicolumn{1}{l|}{$\gamma=\pi / 3, \operatorname{Pr}=0.71, R=1$} & \multicolumn{2}{l|}{$\gamma=\pi / 5, \operatorname{Pr}=0.71, R=1$} \\
\cline { 2 - 7 } & $A=0$ & $A=6$ & $A=0$ & $A=6$ & $A=0$ & $A=6$ \\
\hline$\lambda=0.1$ & 0.42212903 & 0.39032686 & 0.42198201 & 0.38893519 & 0.42174356 & 0.38604911 \\
\hline$\lambda=0.2$ & 0.42725472 & 0.40103726 & 0.42669642 & 0.39823603 & 0.42578501 & 0.39243186 \\
\hline$\lambda=0.5$ & 0.44683000 & 0.43351204 & 0.44389556 & 0.42642715 & 0.43895984 & 0.41174984 \\
\hline$\lambda=2$ & 0.58059870 & 0.59321118 & 0.55921113 & 0.56653749 & 0.51801292 & 0.50937384 \\
\hline$\lambda=3$ & 0.67204447 & 0.68963930 & 0.63992985 & 0.65310971 & 0.57452415 & 0.57253794 \\
\hline
\end{tabular}

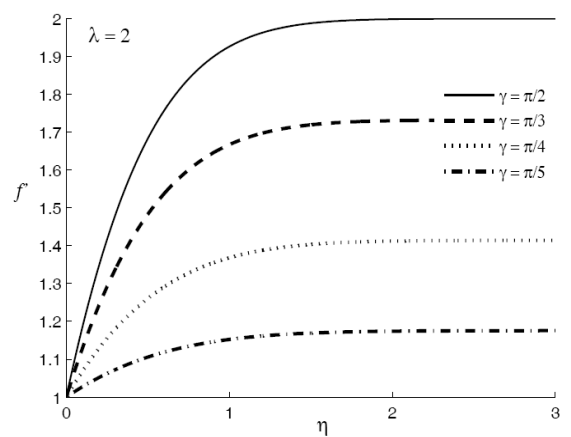

Fig. 4. Velocity profile $f^{\prime}(\eta)$ versus $\eta$ for different values $\gamma$ taking $\lambda=2$ and $A=0$.

friction. Values of the stagnation point $\xi_{s}$ for the favorable flow are given in Table 4. Since the case of unfavorable flow can be mapped to a case of favorable flow by $\gamma \rightarrow \pi-\gamma$ and $\xi \rightarrow-\xi$, we will not present here values of $\xi_{s}$ for the case of unfavorable flow. It has been observed from Table 4 that stagnation point shifts towards origin as striking angle increased from $\pi / 5$ to $\pi / 3$ for any value of $\lambda$. Stagnation point shifts towards origin as porosity parameter increased, and moves to opposite side of the origin, if porosity parameter is further increased. Effect of striking angle $\gamma$ on flow has been represented in Fig. 4. It is found that boundary layer thickness increase as striking angle increases for favourable flow. Figs. 5 and 6 present the velocity profiles for various values of porosity parameter for $\lambda=0.1$ and 2 , respectively. The effect of porosity parameter on velocity profile depends on stretching parameter. Figure 5 shows that the boundary layer thickness decrease considerably as porosity parameter increases for stretching parameter less than one. For $\lambda<1$, the free stream velocity less than stretching velocity and as porosity parameter is increases implies increase in pressure and straining motion near stagnation point which results in thinning of velocity boundary layer. Whereas for $\lambda>1$, velocity boundary layer thickness increase as porosity parameter increases. This is due to inverted boundary layer formed for $\lambda>1$.

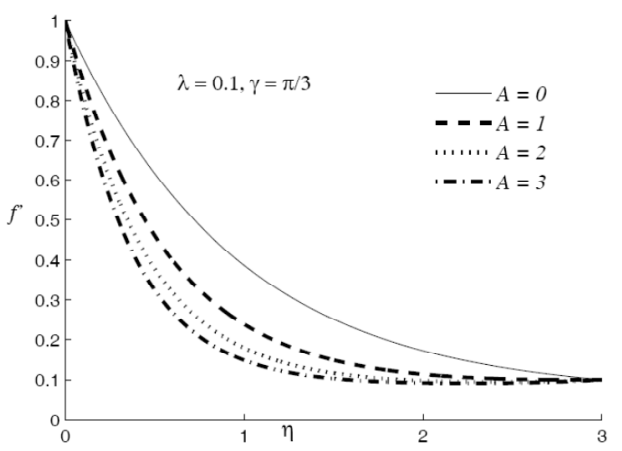

Fig. 5. Velocity profile $f^{\prime}(\eta)$ versus $\eta$ for different values $A$ taking $\lambda=0.1$ and $\gamma=\pi / 3$.

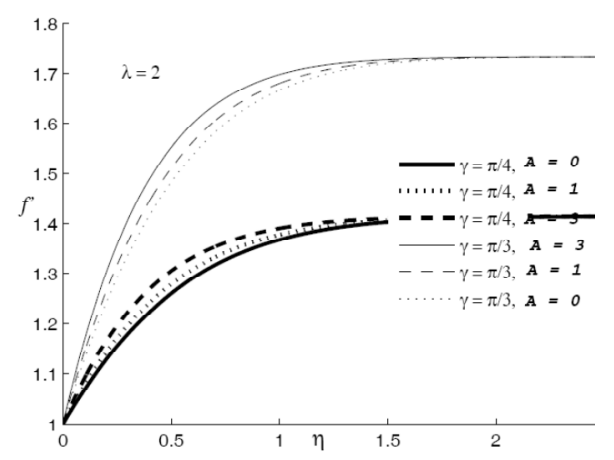

Fig. 6. Velocity profile $f^{\prime}(\eta)$ versus $\eta$ for different values $A$ taking $\lambda=2$ and $\gamma=\pi / 3, \pi / 4$. 
Table 6. Values of rate of heat transfer for different values of stretching sheet parameter $\lambda$, Prandtl number Pr, striking angle $\gamma$ taking radiation parameter $R=1, A=0$.

\begin{tabular}{|l|l|l|l|l|l|l|}
\hline \multicolumn{7}{|c|}{ Value of $-\theta^{\prime}(0)$ for different $\lambda, \operatorname{Pr}, \gamma$} \\
\hline $\begin{array}{l}\text { Value of } \\
\operatorname{Pr}\end{array}$ & $\gamma=\pi / 2, R=1, A=0$ & \multicolumn{1}{l|}{$\gamma=\pi / 3, R=1, A=0$} & \multicolumn{2}{l|}{$\gamma=\pi / 5, R=1, A=0$} \\
\cline { 2 - 7 } & $\lambda=0.1$ & $\lambda=2$ & $\lambda=0.1$ & $\lambda=2$ & $\lambda=0.1$ & $\lambda=2$ \\
\hline $\operatorname{Pr}=0.01$ & 0.3345341 & 0.3369292 & 0.3345321 & 0.33658927 & 0.33452890 & 0.3359544 \\
\hline $\operatorname{Pr}=0.71$ & 0.4221290 & 0.5805987 & 0.4219820 & 0.55921113 & 0.42174356 & 0.5180129 \\
\hline $\operatorname{Pr}=2$ & 0.5929923 & 0.9121825 & 0.5926033 & 0.87422927 & 0.59197193 & 0.7969073 \\
\hline $\operatorname{Pr}=5$ & 0.9655322 & 1.3865492 & 0.9648970 & 1.33556688 & 0.96386461 & 1.2312005 \\
\hline
\end{tabular}

Table 7. Values of rate of heat transfer for different values of stretching sheet parameter $\lambda$, radiation parameter $R$, striking angle $\gamma$ taking Prandtl number $\operatorname{Pr}=0.71$ and $A=0$.

\begin{tabular}{|l|l|l|l|l|l|c|}
\hline \multicolumn{7}{|c|}{ Value of $-\theta^{\prime}(0)$ for different $\lambda, R$ and $\gamma$} \\
\hline \multirow{2}{*}{$\begin{array}{l}\text { Value of } \\
R\end{array}$} & $\gamma=\pi / 2, \operatorname{Pr}=0.71, A=0$ & $\gamma=\pi / 3, \operatorname{Pr}=0.71, A=0$ & \multicolumn{2}{l|}{$\gamma=\pi / 5, \operatorname{Pr}=0.71, A=0$} \\
\cline { 2 - 7 } & $\lambda=0.1$ & $\lambda=2$ & $\lambda=0.1$ & $\lambda=2$ & $\lambda=0.1$ & $\lambda=2$ \\
\hline$R=1$ & 0.42212903 & 0.58059870 & 0.42198201 & 0.55921113 & 0.42174356 & 0.51801292 \\
\hline$R=2$ & 0.38910117 & 0.49477158 & 0.38900808 & 0.48004844 & 0.38885710 & 0.45218450 \\
\hline$R=5$ & 0.35963757 & 0.41139940 & 0.35959352 & 0.40405250 & 0.35952209 & 0.39030890 \\
\hline$R=10$ & 0.34731237 & 0.37507536 & 0.34728896 & 0.37112535 & 0.34725100 & 0.36375190 \\
\hline
\end{tabular}

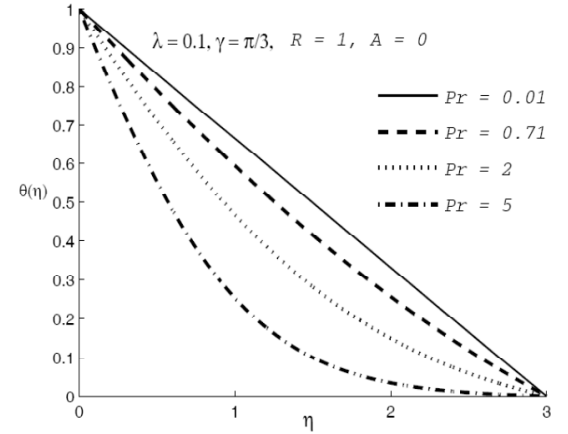

Fig. 7. Temperature profile $\theta(\eta)$ versus similarity variable $\eta$ at different values of Prandtl number $\operatorname{Pr}$ taking $\lambda=0.1, \gamma=\pi / 3, R=1$ and $A=0$.

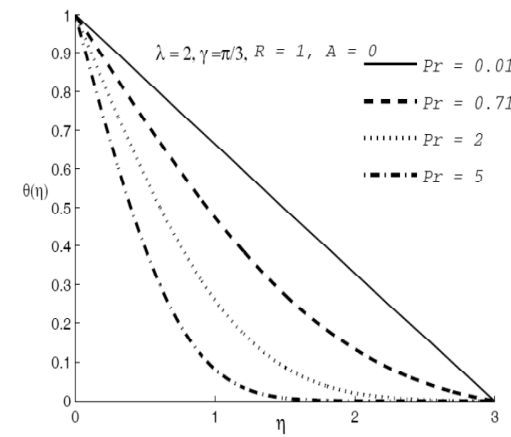

Fig. 8. Temperature profile $\theta(\eta)$ versus similarity variable $\eta$ at different values of Prandtl number $\operatorname{Pr}$ taking $\lambda=2, \gamma=\pi / 3, R=1$ and $A=0$.

It is observed from Table 5 that temperature gradient increase as porosity parameter increases. It is observed from Figs. 7 and 8, and Table 6 that the temperature profile decreases with an increase in the Prandtl number Pr for striking angle $\gamma=\pi / 3$.
This is in agreement with the physical fact that at higher Prandtl number, fluid has a thinner thermal boundary layer and this increases the gradient of temperature. Prandtl number does not depend on stretching sheet parameter.

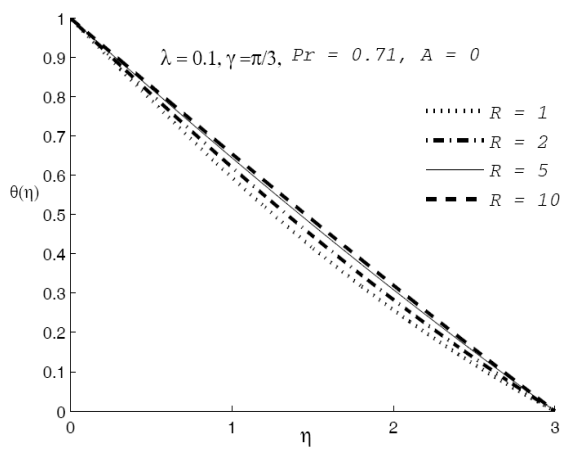

Fig. 9. Temperature profile $\theta(\eta)$ versus similarity variable $\eta$ at different values o radiation parameter $R$ taking $\lambda=0.1, \gamma=\pi / 3$ and $A=0$.

On the other hand, it is also observed from Figs. 9 and 10, and Table 7 that increase of radiation parameter leads to increase in the temperature. This result can be explained by the fact that increase in the values of $R$ for a given of $T_{\infty}$ means a decrease in the Rosseland radiation absorptivity $k_{1}$. The divergence of the radiative heat flux $\partial q_{r} / \partial y$ increases as $k_{1}$ decreases which in turn increases the rate of radiative heat transferred to the fluid and hence the fluid temperature increases. In view of this explanation, the effect of radiation becomes more significant as $R \rightarrow \infty$ and can be neglected when $R \rightarrow 0$. Also, it is seen from figures that the for small value of $R$, the thermal boundary layer thickness is thinner. Therefore higher value of radiation parameter implies higher surface heat flux. 


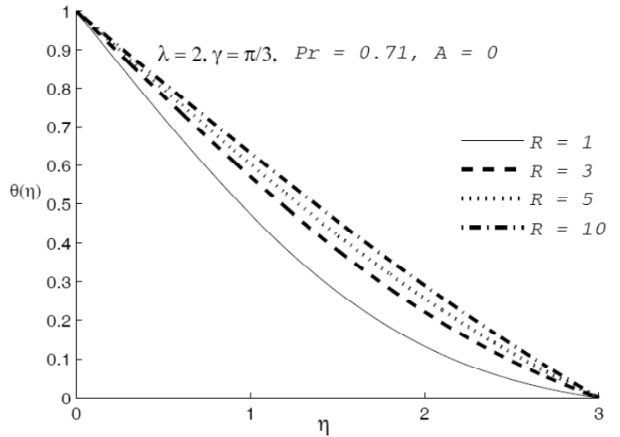

Fig. 10. Temperature profile $\theta(\eta)$ versus similarity variable $\eta$ at different values of radiation parameter $R$ taking $\lambda=2, \gamma=\pi / 3$ and $A=0$.

Attia (2007), Elbashbeshy and Bazid (2009), Anjali Devi and Ganga (2008), Hayat et al. (2010) and Pal and Modal (2010) studies stagnation point flow for porous medium. All the above mention studies consider orthogonal flow only whereas flow can impinge the surface at any angle. We have investigated nonorthogonal flow for above studied parameters. These results have possible technological applications in liquid-based systems involving stretching materials.

\section{Conclusion}

The two dimensional stagnation point flow through a porous medium of a viscous incompressible fluid impinging on a stretching sheet at some angle is studied. A numerical solution for the governing equations is obtained which allows the computation of the flow and heat transfer characteristics for various values of the porosity parameter, the stretching sheet parameter, striking angle, radiation parameter and the Prandtl number. The main results of the paper can be summarized as follows:

1) The boundary layer thickness decreases as stretching parameter increases.

2) Stagnation point shifts towards origin as striking angle increases.

3) Boundary layer thickness increases as striking angle increases.

4) Effects of porosity parameter depend on stretching parameter.

5) Temperature gradient increases as porosity parameter increases.

6) Temperature decreases with increase in the Prandtl number.

7) Radiation parameter increases with increase of the temperature.

\section{REFERENCES}

Amaouche, M. and D. Boukari (2003). Influence of thermal convection on non-orthogonal stagnation point flow. International Journal of Thermal Sciences 42, 303-310.

Anjali Devi, S.P. and B. Ganga (2008). Dissipation effects on MHD nonlinear flow and heat transfer past a porous surface with prescribed heat flux. Journal of Applied Fluid Mechanics 3, 1-6.
Attia, H.A. (2007). On the effectiveness of porosity on unsteady flow between parallel plates of a viscoelastic fluid under constant pressure gradient with heat transfer. Computational Materials Science 38, 746-750.

Attia, H.A. (2007). On the effectiveness of porosity on stagnation point flow towards a stretching surface with heat generation. Computational Materials Science 38, 741-745.

Crane, L.J. (1970). Flow past a stretching plate. J. Appl. Math. Phys. (ZAMP) 21, 645-647.

El-Aziz, M.A. (2009). Radiation effect on the flow and heat transfer over an unsteady stretching sheet. International Communications in Heat and Mass Transfer 36, 521-524.

Elbashbeshy E.M.A. and M.A.A. Bazid (2004). Heat transfer in a porous medium over a stretching surface with internal heat generation and suction or injection. Applied Mathematics and Computation 158, 799-807.

Ghaly, A.Y. and E.M.E. Elbarbary (2002). Radiation effect on MHD free -convection flow of a gas at a stretching surface with a uniform free stream. Journal of Applied Mathematics 2, 93-103.

Gupta, A.S. and T.R. Mahapatra (2003). Stagnationpoint flow towards a stretching surface. The Canadian Journal of Chemical Engineering 81, 258-263.

Hayat, T., Z. Abbas, I. Pop and S. Asghar (2010). Effects of radiation and magnetic field on the mixed convection stagnation-point flow over a vertical stretching sheet in a porous medium. International Journal of Heat and Mass Transfer $53,466-474$.

Labropulu, F., D. Li and I. Pop (2010). Non-orthogonal stagnation-point flow towards a stretching surface in a non-Newtonian fluid with heat transfer. International Journal Thermal Sciences 49, 10421050.

Li, D., F. Labropulu and I. Pop (2009). Oblique stagnation-point flow of a viscoelastic fluid with heat transfer. International Journal of Non-Linear Mechanics 44, 1024 - 1030

Lok, Y.Y., N. Amin and I. Pop (2007). Mixed convection flow near a non-orthogonal stagnation point towards a stretching vertical plate. International Journal of Heat and Mass Transfer 50, 4855-4863.

Lok, Y.Y., N. Amin and I. Pop (2007). Comments on:“ Steady two-dimensional oblique stagnationpoint flow towards a stretching surface": M. Reza and A.S. Gupta, Fluid Dynamics Research 37 (2005) 334-340. Fluid Dynamics Research 39, 505-510. 
Lok, Y.Y., I. Pop and A.J. Chamkha (2007). Nonorthogonal stagnation-point flow of a micro polar fluid. International Journal of Engineering Science 45, 173-184.

Lok, Y.Y., N. Amin and I. Pop (2006). Nonorthogonal stagnation point flow towards a stretching sheet. International Journal of NonLinear Mechanics 41, 622-627.

Lok, Y.Y., N. Amin and I. Pop (2006). Mixed convection near a non-orthogonal stagnation point flow on a vertical plate with uniform surface heat flux. Acta Mechanica 186, 99-112.

Pal, D. and H. Mondal (2010). Effect of variable viscosity on MHD non-Darcy mixed convective heat transfer over a stretching sheet embedded in a porous medium with non-uniform heat source/sink. Commun Nonlinear Sci Numer Simulat 15, 1553-1564.

Pop, I., S.R. Pop and T. Grosan (2004). Radiation effects on the flow near the stagnation point. Technische Mechanik 25, 100-106.

Reza, M. and A.S. Gupta (2005). Steady twodimensional oblique stagnation-point flow towards a stretching surface. Fluid Dynamics Research 37, 334-340.

Rosali, H., A. Ishak and I. Pop (2011). Stagnation point flow and heat transfer over a stretching/shrinking sheet in a porous medium. International Communications in Heat and Mass Transfer 38, 1029-1032.

Singh, P., N.S. Tomer, S. Kumar and D. Sinha (2011). Effect of Radiation and Porosity Parameter on Magnetohydrodynamic Flow due to Stretching Sheet in Porous Media. Thermal Sciences 15(2), 517-526.

Sharma, P.R. and G. Singh (2009). Effects of variable thermal conductivity and heat source/sink on MHD flow near a stagnation point on a linearly stretching sheet. Journal of Applied Fluid Mechanics 2, 13-21.

Tilley, B.S. and P.D. Weidman (1998). Oblique twofluid stagnation-point flow. European Journal of Mechanics 17, 205-217. 